\title{
The neural features in the precentral gyrus predict the severity of internet game disorder: results from the multi-voxel pattern analyses
}

\author{
Shuer Ye a, Msc; Min Wang a,b,c, Msc; Qun Yang a*, PhD; Haohao Dong d, Msc; Guang-Heng Dong \\ a,b,c*, PhD \\ a Institute of Psychological Science, Hangzhou Normal University, Hangzhou, P.R. China \\ b Center for Cognition and Brain Disorders, the Affiliated Hospital of Hangzhou Normal University, \\ Hangzhou, P.R. China \\ c Zhejiang Key Laboratory for Research in Assessment of Cognitive Impairments, Hangzhou, Zhejiang \\ Province, P.R. China \\ d Department of Psychology, Zhejiang Normal University, Jinhua, P.R. China
}

\section{Corresponding Authors:}

\section{Qun Yang, Ph.D.}

Institute of Psychological Science, Hangzhou Normal University, 2318 Yuhangtang Road, Hangzhou, P.R. China.

Tel.: +861358870011.

E-Mail: qunyang@hznu.edu.cn

\section{Guang-Heng Dong, Ph.D., Professor}

Center for Cognition and Brain Disorders, The Affiliated Hospital of Hangzhou Normal University, 2318 Yuhangtang Road, Hangzhou, Zhejiang Province, P.R. China.

Tel.: +86 15867949909 .

E-Mail: dongguangheng@hznu.edu.cn

\section{Manuscript Information}

Number of pages: 27

Number of figures and tables: $4 / 2$

Number of words for Introduction/Method/Results/Discussion: 1,063/1,647/386/1,104; (4200 total) 


\section{Key Points}

Question: Can machine learning algorithms predict internet gaming disorder (IGD) from resting-state neural patterns?

Findings: This diagnostic study collected resting-state fMRI data from 402 subjects with diverse IGD severity. We found that machine learning models based on resting-state neural patterns yielded significant predictions of IGD severity. In addition, the topological neural features of precentral gyrus, which is a consensus highly weighted region, is significantly correlated with IGD severity.

Meaning: The study found that IGD is a distinctive disorder and its dependence severity could be predicted by brain features. The precentral gyrus and its connection with other brain regions could be view as targets for potential IGD intervention, especially using brain modulation methods. 


\section{Abstract}

Importance: Finding the neural features that could predict internet gaming disorder severity is important

in finding the targets for potential interventions using brain modulation methods.

Objective: To determine whether resting-state neural patterns can predict individual variations of internet gaming disorder by applying machine learning method and further investigate brain regions strongly related to IGD severity.

Design: The diagnostic study lasted from December 1, 2013, to November 20, 2019. The data were analyzed from December 31, 2019, to July 10, 2020.

Setting: The resting-state fMRI data were collected at East China Normal University, Shanghai.

Participants: A convenience sample consisting of 402 college students with diverse IGD severity

Main Outcomes and Measures: The neural patterns were represented by regional homogeneity (ReHo) and the amplitude of low-frequency fluctuation (ALFF). Predictive model performance was assessed by Pearson correlation coefficient and standard mean squared error between the predicted and true IGD severity. The correlations between IGD severity and topological features (i.e., degree centrality (DC), betweenness centrality (BC), and nodal efficiency (NE)) of consensus highly weighted regions in predictive models were examined.

Results: The final dataset consists of 402 college students (mean [SD] age, 21.43 [2.44] years; 239 [59.5\%] male). The predictive models could significantly predict IGD severity (model based on ReHo: $r=0.11, p(r)$ $=0.030, \mathrm{SMSE}=3.73, p(\mathrm{SMSE})=0.033 ;$ model based on ALFF: $r=0.19, p(r)=0.002, \mathrm{SMSE}=3.58, p(\mathrm{SMSE})$ $=0.002)$. The highly weighted brain regions that contributed to both predictive models were the right precentral gyrus and the left postcentral gyrus. Moreover, the topological properties of the right precentral gyrus were significantly correlated with IGD severity (DC: $r=0.16, p=0.001$; BC: $r=0.14, p=0.005$; NE: $r$ 
$=0.15, p=0.003$ ) whereas no significant result was found for the left postcentral gyrus (DC: $r=0.02, p=$

0.673; BC: $r=0.04, \mathrm{p}=0.432$; NE: $r=0.02, p=0.664$ ).

Conclusions and Relevance: The machine learning models could significantly predict IGD severity from

resting-state neural patterns at the individual level. The predictions of IGD severity deepen our

understanding of the neural mechanism of IGD and have implications for clinical diagnosis of IGD. In

addition, we propose precentral gyrus as a potential target for physiological treatment interventions for

IGD. 


\section{Introduction}

Internet gaming disorder (IGD) has been recognized as a serious mental health problem that is

characterized by obsession with gaming, hypersensitivity to game cues and failure to resist the impulse to

play games despite negative consequences ${ }^{1-3}$. In recent decades, there has been a steady increase in IGD

around the world, and an increased number of studies have focused on behavioral characteristics

associated with IGD ${ }^{4-6}$. In 2013, IGD was included in Section III of the DSM-5 (The Diagnostic and

Statistical Manual of Mental Disorders, Fifth Edition) as a condition requiring future research ${ }^{7}$. In 2015,

gaming disorder was officially listed in the new version of the International Classification of Diseases

(https://www.who.int/news-room/q-a-detail/gaming-disorder). Despite widespread interest in IGD, less

is known about the neural substrates underlying this disorder.

The behavioral model of IGD has been well developed thus far. Extreme reward-seeking ${ }^{8}$, defective

executive inhibition ${ }^{9}$, and risky decision-making ${ }^{10}$ are commonly recognized as core components of IGD ${ }^{5}$,

11. These findings provide a basis for clinical behavior therapy for IGD ${ }^{12,13}$. However, most of these

cross-sectional studies revealed the static con dition of IGD, which cannot explain the relationship between

neural features and the degree of addiction. Thus, the exploration of neural substrates of IGD may advance

our understanding of this mental disorder and provide new insights for diagnosis, intervention, and

treatment.

Resting-state functional magnetic resonance imaging (rs-fMRI) is an effective task-independent

method used to investigate the neural substrates of IGD. Two metrics of rs-fMRI were developed to

measure neural activities in the human brain: (i) regional homogeneity (ReHo) measures regional

synchronization at the whole-brain level and spontaneous local neural activity ${ }^{14}$, and (ii) the amplitude of

low-frequency fluctuation (ALFF) measures regional intensity of spontaneous fluctuations in the BOLD 
signal. These two neural indicators have been effectively used to explore the neural mechanism of IGD 15-17.

For instance, ReHo alterations were found in regions related to sensory-motor coordination, audiovisual

processing, and reward pathways in individuals with IGD compared with healthy controls ${ }^{18}$. Additionally,

studies that employed the ALFF method found abnormalities in the left medial orbitofrontal cortex, the left

precuneus, the left supplementary motor area, the right parahippocampal gyrus, and the bilateral middle

cingulate cortex among individuals with IGD ${ }^{19}, 20$. Therefore, IGD has been widely proven to be related to

abnormal brain activity, which leads to its addiction pattern ${ }^{21-23}$. However, few studies have had a sample

size large enough to ensure the reliability of their findings. More importantly, previous studies using

conventional fMRI analyses were unable to effectively utilize massive fMRI data to delineate neural

patterns and often focused on the differences between individuals with IGD and normal individuals at the

group level.

Multivoxel pattern analysis (MVPA), a powerful data-driven machine learning method, is widely used

in decoding brain activities and providing useful neural information to improve mental disorder diagnosis

and treatment ${ }^{24,25}$. MVPA has unique advantages (e.g., having an increased sensitivity to detect brain

patterns, allowing the extraction of feature weights, and characterizing neural code at the individual level)

compared with conventional fMRI analyses. This method has been implemented to characterize neural

coding and information processing in psychiatric studies ${ }^{26,27}$ and could be useful in identifying the neural

features of IGD. However, to date, only one study has explored the neurobiological mechanism of IGD by

using MVPA ${ }^{28}$, suggesting that MVPA provides a potential way to distinguish individuals with IGD from

recreational game users by decoding brain patterns represented by ReHo values. However, whether

multiple brain activities can predict the IGD features of an individual remains unknown. Thus, determining

whether different measures can predict IGD features and how they work is important and necessary. 
Studies have demonstrated that addiction is related not only to abnormal brain activities in a specific region but also to atypical interactions between brain regions and networks ${ }^{22,29}$. Altered functional connectivity was shown between regions involving reward, cognitive processing, and executive control function in subjects with IGD 30,31. Although MVPA based on local neural features can characterize brain patterns, it cannot be used to explore the regional relationships involved in IGD. Thus, graph theory and Granger causality analysis (GCA) were applied to address this problem. Graph theory provides a powerful framework to describe the whole brain topologically and has been widely used in studies about addiction 32. By constructing a network made up of nodes and edges, several topological metrics can be calculated to characterize regional properties and then reveal the potential significant position of the regions in the brain. Wang et al. ${ }^{33}$ reported that subjects with IGD showed reduced node metrics in executive control and emotion-related regions, indicating the key roles these regions played in IGD. In addition, Granger causality analysis, which is a mathematical method used to build effective connectivity, can be applied to detect coupling among regions without assumptions about connections between them ${ }^{34,35}$. To date, two task fMRI studies have used GCA to explore the neural substrates of IGD. One study found that IGD severity was negatively correlated with connectivity from the middle frontal gyrus to the precuneus during a cue-carving task, and another study found abnormal effective connectivity within the salience network in adolescents with IGD ${ }^{36,37}$.

In the present study, we aimed to combine MVPA and graph theory analysis with GCA to decode the specific neural patterns of IGD in a large sample. We first applied the MVPA method to identify highly weighted regions as core brain areas in predicting IGD. The subsequent analysis revealed how these crucial regions for IGD work and interact with other regions. Specifically, we used MVPA to examine whether ReHo and ALFF could predict individuals' IGD severity and to identify relatively highly weight 
brain regions that contributed to the model. Then, graph theory analysis was employed to further confirm the importance of selected regions in the IGD brain networks. Finally, GCA was implemented to explore how these regions interact with other brain regions in contributing to IGD. Based on previous studies, we hypothesized that (1) the predictive model based on ReHo and ALFF could significantly predict IGD severity; (2) regions related to reward processing, sensory-motor coordination, and executive control would be highly weighted in prediction models; (3) these highly weighted regions are crucial in the IGD brain network; and (4) IGD severity is related to effective connectivity between these regions and other regions in the whole brain. 


\section{Methods}

\section{Participants}

Four hundred and two right-handed participants ( 239 males; $21.43 \pm 2.44$ years old) were recruited by advertisement from June 2013 to December 2019. All participants were free of any personal or family history of psychiatric disorders as assessed by an exhaustive structured psychiatric interview. Participants' demographics and internet addiction test (IAT) scores are shown in Tab. 1. The study was conducted in accordance with the 1964 Helsinki Declaration and its later amendments and was approved by the Ethics Committee of Zhejiang Normal University. Written informed consent was obtained from all participants before participating in this research.

\section{----- Insert Table 1 about here ------}

\section{Measures}

Participants were assessed for IGD severity with Young's online Internet Addiction Test (IAT) ${ }^{38}$. The IAT consists of 20 items related to internet use and internet-related addictive behavior. Each item can be rated on a 5-point Likert scale (from 1-rarely to 5-always). Higher IAT scores indicate greater internet use and addiction. The reliability and validity of the scale have been well validated ${ }^{39}, 40$. In addition to the IAT, the DSM-5, which is a nine-item IGD diagnostic measure, was also applied to assess IGD severity ${ }^{2}$. Participants were asked to answer "yes" or "no" to nine criteria occurring over the past 12 months. People who met more than 5 criteria were clinically diagnosed with IGD. Because the DSM-5 was published in 2014, only part of our participants $(\mathrm{N}=365)$ took the assessment. IAT scores and DSM-5 scores are highly correlated.

\section{MRI data acquisition}

Resting-state functional magnetic images were collected at East China Normal University using a 3T MRI system (Siemens Trio). The participants were simply instructed to keep their eyes closed and stay awake 
without performing any cognitive exercises. Head motion was minimized using foam padding and

restraint. The imaging parameters were as follows: repetition time $(\mathrm{TR})=2000 \mathrm{~ms}$, interleaved 33 slices,

echo time $(\mathrm{TE})=30 \mathrm{~ms}$, thickness $=3.0 \mathrm{~mm}$, flip angle $=90^{\circ}$, field of view $=220 \times 220 \mathrm{~mm}$, and matrix $=64$

$\times 64$. Each fMRI scan lasted for $420 \mathrm{~s}$ and included 210 imaging volumes.

\section{Preprocessing}

Preprocessing was conducted with DPABI v3.0 (Data Processing \& Analysis for Brain Imaging:

http://rfmri.org/dpabi), which is a pipeline toolbox for fMRI analysis in MATLAB ${ }^{41}$. For each participant, the first 10 volumes were discarded to minimize the (transient signal) instability of the initial signal and adapt participants to the scanning environment (effect of scanner calibration). Subsequent data preprocessing included slice timing correction, head motion correction, spatial normalization to the standard MNI space with an EPI template and resampling into $3 \times 3 \times 3 \mathrm{~mm}^{3}$ voxels. The data used in the present study met the criteria of head motion $<2.5 \mathrm{~mm}$ or $2.5^{\circ}$. Nuisance signals, including 24 motion vectors (i.e., six 6 head motion parameters, 6 head motion parameters one time point before, and the 12 corresponding squared items), the white matter signal, and the cerebrospinal fluid signal, were regressed out ${ }^{42}$. Subsequently, the linear trends of time courses were removed, and the resulting images were temporally filtered with a bandpass filter $(0.01-0.1 \mathrm{~Hz})$ to reduce the effect of low frequency drift and high-frequency noise ${ }^{43}$. Finally, the images were spatially smoothed using a Gaussian filter to decrease spatial noise $\left(6 \times 6 \times 6 \mathrm{~mm}^{3}\right.$ full width at half maximum $)$.

\section{MVPA}

\section{ReHo and ALFF calculation}

The ReHo and ALFF map of each subject was calculated with DPABI to evaluate local spontaneous activity and brain functional synchronization in the resting state. The ReHo map of each subject was generated by 
calculating Kendall's coefficient of concordance between a single voxel and the 26 nearest neighbor voxels in a voxel-wise manner for the entire time series ${ }^{14}$. Note that the ReHo calculation used rs-fMRI data that were not smoothed during preprocessing. The ALFF map of each subject was generated using the following steps. First, the imaging data were temporally bandpass filtered $(0.01<\mathrm{f}<0.08 \mathrm{~Hz})$. Second, the time series of each voxel was transformed into the frequency domain to obtain the power spectrum, and the square root was calculated at each frequency of the power spectrum. Finally, the average square root was obtained across $0.01-0.08 \mathrm{~Hz}$ at each voxel and then taken as the ALFF value ${ }^{44}$.

\section{Kernel ridge regression (KRR)}

Using the ReHo and ALFF maps, MVPA was implemented to predict IGD severity (i.e., IAT score) in the Pattern Recognition for Neuroimaging Toolbox (PRoNTo: http://www.mlnl.cs.ucl.ac.uk/pronto) ${ }^{45}$. The

KRR was utilized as the regression algorithm in the present study. KRR, which is a kernel-based approach, has very good generalization performance. The linear kernel method was used to map these implicit features into a high dimensional feature space ${ }^{46}$. Then, ridge regression, a linear least square regression with Tikhonov regularization (regularization that penalizes the sum of squares of the weights) was applied to predict the IGD severity ${ }^{47,48}$. Here, the ReHo and ALFF maps were used as input data in the analyses. Each 3D image was transformed into a column vector of features, and each value corresponded to a single corresponding voxel intensity. Then, whole-brain models with features from images were constructed to investigate whether the ReHo/ALFF pattern could predict IGD severity.

\section{Cross-validation method}

To test the generalizability of the predictve models, leave-one-out cross-validation (LOOCV) was

implemented. The method involved all subjects training the model, but one was left out to obtain an estimated model. The model was used to predict the behavior of the left-out subject. The above 
procedures were repeated $\mathrm{n}$ times ( $\mathrm{n}=$ total number of subjects) to obtain a relatively unbiased estimate of generalizability. The performance of the established model was evaluated by two metrics, Pearson's correlation coefficient $(r)$ and the standard mean squared error (SMSE) ${ }^{49}$. The correlation coefficient refers to the strength of a linear relationship between two variables. A higher correlation indicates better predictions. The SMSE refers to the mean of the squared differences between the predicted and true scores divided by the targets' variance. The significance of these predictions was assessed with a permutation test of 1,000 permutations. That is, the same cross-validation procedure mentioned above was performed 1000 times with the label permuted across all the participants. The number of permutations that showed better performance (i.e., higher $r$ or lower SMSE than the value obtained with the true target) was

calculated. The p-value was computed by dividing the number by the total number of permutations (i.e., 1,000). The workflow for the MVPA is presented in Fig. 1.

\section{----- Insert Figure 1 about here ------}

\section{Region of interest (ROI) selection}

A weighted image can be calculated by PRoNTo voxel-wise and ROI-wise for each model. To find the most weighted region in the whole brain, the Dosenbach 160-node atlas was applied to define the brain regions 45, 50. The ROI contributions to the predictive model were ranked in descending order, and regions that were $5 \%$ of the maximum (i.e., top 8 ) weighted were listed from predictive models that was based on the ReHo and ALFF patterns respectively. Regions that were presented in both top 8 lists were selected as ROIs in the current study.

\section{Graph theory measures}

To test the significance of these ROIs in IGD severity across the brain network, graph theory was employed to characterize the topological properties of the ROIs. In graph theory, a topological brain network can be 
constructed by nodes and edges. Nodes refer to brain regions predefined by an atlas, and edges are defined as functional connectivity between two regions using Pearson's correlation coefficient. Here, three nodal properties were computed to describe ROIs we selected in the predictive model. The degree centrality (DC) is the number of edges connected to a given node; it quantifies the information communication ability of nodes in the network ${ }^{51}$. The betweenness centrality (BC) is the number of shortest paths passing through a given node and describes the effect of the node on the information transmission of other nodes ${ }^{52}$. The nodal efficiency (NE) is the average inverse shortest path length between the given node and every other node, and it characterizes the efficiency of parallel information transfer by the node ${ }^{53}$.

The nodes were defined using Dosenbach's 160-node atlas, which was employed in the MVPA above. The edges were the functional connectivity between each pair of nodes, computed as Pearson's correlation between the time courses of each pair of ROIs. First, the preprocessed data were entered to calculate correlation coefficients between each pair of ROIs, and subsequent correlation coefficients were normalized to $\mathrm{Z}$-scores with Fisher's r-to-Z transformation. Thus, a weighted undirected functional connectivity matrix was generated, and it was converted to a graph network by considering a threshold $T$ (set as 0.25 in the present study) to ensure that the stronger edges, in descending order, can enter the network construction ${ }^{54}$. Then, the weighted network was binarized, so that $\mathrm{DC}$, BC, and NE could be calculated for every node in the setting threshold. Finally, correlation analysis was conducted between these topological metrics of the ROIs and IGD severity. The procedures mentioned above were implemented in the GRaph thEoreTical Network Analysis toolbox (GRETNA: http://www.nitrc.org/projects/gretna// ${ }^{55}$.

\section{Granger Causality Analysis}


Although GCA was originally developed in the field of economics to find the causal relationship between two time-courses, it has also been widely applied in neuroscience studies. Here, voxel-wise GCA was employed to evaluate effective connectivity related to selected ROIs. DynamicBC, a MATLAB toolbox, allows performance of GCA for rest-state fMRI data ${ }^{56}$. The preprocessed $4 \mathrm{D}$ rest-state fMRI image was entered to calculate effective connectivity between ROIs and voxels within the whole brain. Thus, IN (i.e., information transmitted from other voxels to a given ROI) and OUT (i.e., information transmitted from a given ROI to other voxels) effective connectivity brain maps were generated separately for selected ROIs in each subject. Correlation analyses were conducted using IGD severity to determine how information flow through selected ROIs was influenced by IGD severity. 


\section{Results}

\section{MVPA results}

The two predictive models (ReHo and ALFF) yielded similar and significant predictions of IGD severity (model based on ReHo: $r=0.11, p(r)=0.030$, SMSE=3.73, $p$ (SMSE) $=0.033$; model based on ALFF: $r=0.19$, $p(r)=0.002, \mathrm{SMSE}=3.58, p(\mathrm{SMSE})=0.002)$

\section{----- Insert Figure 2 about here ------}

For each model, the region weights were ranked in descending order. We found two regions, namely, the right precentral gyrus $(60,8,34, x, y, z)$ and left postcentral gyrus $(-54,-9,23, x, y, z)$, were shown in the top 8 predictor lists of the two models and were therefore chosen as our ROIs.

\section{Graph theory analysis results}

Graph theory analysis was applied to identify the important role of these highly weighted ROIs in IGD brain networks. Significant positive associations were found between IGD severity and all three graph theory metrics (i.e., DC, BC, and ND) of the right precentral gyrus (DC: $r=0.16, p=0.001$; $\mathrm{BC}: r=0.14, p=$ 0.005; NE: $r=0.15, p=0.003$ ), whereas no significant result was found for the left postcentral gyrus (DC: $r$ $=0.02, p=0.673$; BC: $r=0.04, p=0.432$;E: $r=0.02, p=0.664$ ) (Fig. 3). These results indicated that the precentral gyrus may play a significant role in the whole-brain network. We reasoned that the precentral gyrus works as an information intermediary responsible for transmitting and integrating information in an IGD brain. To define these potential pathways, GCA was implemented using the precentral gyrus as our seed region.

\section{----- Insert Figure 3 about here ------}

\section{GCA results}

Finally, GCA was applied to investigate the interactions between the right precentral gyrus and the whole 
brain. When correlating IGD severity (IAT score) with the effective connectivity results (input/output from

right precentral gyrus), two effective connectivity that output from the right precentral gyrus to the left

precentral gyrus (cluster size: 99 voxels; MNI coordinates: 33, -9, 57, x, y, z) and dACC (cluster size: 63

voxels; MNI coordinates: $-9,12,45, \mathrm{x}, \mathrm{y}, \mathrm{z})$ were positively correlated with IAT score $(p<0.005$, GRF

corrected) (Fig. 4).

\section{----- Insert Figure 4 about here ------}

We reported the results using IAT score in selecting IGD, the results are of similar when using DSM-5

scores in selecting IGD. All results using DSM-5 scores were put into the supplementary materials. 


\section{Discussion}

In the present study, we combined machine learning techniques with rs-fMRI to identify distinct neural patterns in predicting IGD severity. The results showed that neural models represented by both ReHo and ALFF could significantly predict IGD severity at the individual level. In particular, the right precentral gyrus and left postcentral gyrus were highly weighted in both prediction models. Furthermore, graph theory analysis implied that the right precentral gyrus is an important node in the IGD brain network. Finally, GCA revealed that the effective connectivity between the right precentral gyrus and the left precentral gyrus and dACC was related to IGD severity.

\section{Neural patterns detected by MVPA can predict IGD severity}

Previous studies have demonstrated that the resting-state neural patterns underlying IGD fit with its diverse behavioral characteristics. For example, subjects with IGD showed increased ReHo in brain regions involved in sensory-motor coordination ${ }^{18}$. Furthermore, altered fALFF was observed in the cerebellum posterior lobe and superior temporal gyrus, which may be related to cognitive function and movement 57 .

Additionally, a recent meta-analysis found that abnormalities existed in several brain networks, including the default mode network, frontoparietal network, and attention network, in patients with IGD ${ }^{58 .}$

Although these findings advance the understanding of the neural mechanism of IGD, the stability and reproducibility of these results still need to be evaluated with caution. The machine learning approach could decode complex brain patterns of mental disorders voxel-wise, which is more reliable than conventional fMRI analyses ${ }^{24,59}$. Here, we applied the MVPA method with two rs-fMRI metrics-ReHo and AlFF-to predict IGD severity. We found that both ReHo and ALFF can significantly predict addiction severity at the individual level. The results suggested that resting-state brain activities can be used to predict IGD severity effectively and imply the distinctive neural patterns underlying IGD. The brain 
activities in the informative brain regions of the predictive models were accompanied by changes in the severity of IGD, and these regions may play a key and distinct role in IGD. Moreover, consensus highly weighted brain regions were revealed by two predictive models, indicating that the results of MVPA are relatively robust and that neural signatures that can be detected by machine learning methods stably exist in IGD brains.

\section{Neural features in the precentral gyrus play a key role in predicting IGD severity}

In both predictive models, the precentral gyrus and postcentral gyrus were reported as informative seeds for predicting IAT scores. The precentral gyrus and postcentral gyrus are the key regions of sensorimotor networks associated with integrating sensorimotor information and coordinating physical movement 60,61 . Atypical brain activities related to sensorimotor networks have been consistently indicated in IGD individuals previously ${ }^{20}$. For example, IGD subjects showed enhanced ReHo values in brain regions associated with motor-sensory coordination ability and altered functional connectivity in sensory-motor related networks ${ }^{18,33}$. In line with these findings, the precentral gyrus and postcentral gyrus play a key role in motor functions, especially in coordinating hand movements, which is very important for internet game playing. The results of graph theory analysis further revealed the special role of the right precentral gyrus in IGD whole-brain networks. Elevated DC, BC, and NE of the right precentral gyrus were associated with greater IGD severity, indicating that the precentral gyrus may become a functional hub of the brain network that is responsible for information propagation, integration, and processing with increasing IGD severity ${ }^{62,63}$. Moreover, frequent computer game usage improves motor-visual coordination and perceptual-motor competencies ${ }^{64,65}$. Therefore, frequent internet game use, which is one of the features of IGD, may enhance interactions between the precentral gyrus and other regions to proficiently complete operations required by computer games ${ }^{33}$. 


\section{Effective connectivity between the precentral gyrus and dACC related to IGD severity}

Furthermore, we found that effective connectivity from the right precentral gyrus to the left

precentral gyrus and the dACC was positively correlated with IGD severity. The effective connectivity from the right precentral gyrus to the left precentral gyrus may be related to the coordination of sensorimotor information between the left and right brain. Here, the increased effective connectivity between the left and the right precentral gyrus may indicate advanced game skills in individuals with IGD ${ }^{66}$. Moreover, we found increased effective connectivity between the right precentral gyrus and the dACC, which is a node of salience networks in elevated IGD participants. The ACC is primarily associated with response selection ${ }^{67}$, motivation ${ }^{68}$, and reward assessment ${ }^{69}$. The dorsal part of this region (i.e., the dACC) has been especially indicated, by an increasing body of research, to be dysfunctional among individuals with IGD ${ }^{70-72}$. The dysfunction of the dACC may contribute to disadvantageous decision-making and cue-induced carving in addiction behaviors ${ }^{73,74}$. In the current study, we speculated that the pathway from the right precentral gyrus to the dACC may be responsible for transmitting and then integrating sensorimotor information into decision-making and reward evaluation. The enhancement of this pathway among individuals with IGD suggests more frequent communication between the right precentral gyrus and the dACC, leading to rapid reward assessment and risky decision-making ${ }^{75}$. When subjects with IGD are exposed to game cues, they are strongly motivated to be involved in games and quickly respond to these cues, leading to their addiction and risky behavior. The effective connectivity results may reveal a neural circuit existing in the IGD brain. The enhancement of the circuit fits symptoms of IGD, i.e., that individuals are proficient in operating computer games that require extensive sensorimotor coordination and that they exhibit increased reward craving and strong motivation to play internet games 7,33.

\section{Limitations:}


There were some limitations in this study. First, because this study was limited to a population of college students, most of whom are adults, it is difficult to determine whether the results would be the same in teenager samples. Future studies should examine the results in wider sample types. Second, we did not examine other highly weighted regions in the predictive model. Although some regions have a high weight only in a single model, they still have neurophysiological significance for IGD. How these regions' neural activity is related to IGD severity needs full consideration and exploration.

\section{Conclusions}

The current study demonstrated that resting-state neural activity could predict IGD severity at the individual level. As such, the findings may provide evidence to support the view that IGD has specific neural patterns and provide new insight into IGD. In addition, we discovered and verified the critical role of the precentral gyrus in IGD brain networks. The current study deepened our understanding of the neural mechanism of IGD and provides a potential target for physiological treatment interventions for IGD. 


\section{ACKNOWLEDGMENTS}

The current research was supported by the Zhejiang Natural Science Foundation (LY20C090005).

The funding agencies did not have input into the writing of this manuscript.

\section{Authors' contributions}

Shuer Ye wrote the first draft of the manuscript. Shuer Ye and Ming Wang analyzed the data. Haohao

Dong contributed to fMRI data collection. Guang-heng Dong designed this research. Guang-heng Dong and

Qun Yang edited the manuscript. All authors contributed to and approved the final manuscript.

\section{Conflicts of interest}

The authors declare that no competing interests exist. 


\section{References}

1. Brand M, Young KS, Laier C, Wölfling K, Potenza MN. Integrating psychological and neurobiological considerations regarding the development and maintenance of specific Internet-use disorders: An Interaction of Person-Affect-Cognition-Execution (I-PACE) model. Neuroscience Biobehavioral Reviews. 2016;71:252-266.

2. Petry NM, Rehbein F, Gentile DA, et al. An international consensus for assessing internet gaming disorder using the new DSM-5 approach. Addiction. 2014;109(9):1399-1406. doi:10.1111/add.12457

3. Dong G, Li H, Wang L, Potenza MN. Cognitive control and reward/loss processing in Internet gaming disorder: results from a comparison with recreational Internet game-users. European Psychiatry. 2017;44:30-38. doi:10.1016/j.eurpsy.2017.03.004

4. Mihara S, Higuchi S. Cross-sectional and longitudinal epidemiological studies of I nternet gaming disorder: A systematic review of the literature. Psychiatry clinical neurosciences. 2017;71(7):425-444. doi:10.1111/pcn.12532

5. Dong G, Potenza MN. A cognitive-behavioral model of Internet gaming disorder: theoretical underpinnings and clinical implications. Journal of psychiatric research. 2014;58:7-11.

6. Király O, Griffiths MD, Demetrovics Z. Internet gaming disorder and the DSM-5: Conceptualization, debates, and controversies. Current Addiction Reports. 2015;2(3):254-262.

7. Petry NM, O'Brien CP. Internet gaming disorder and the DSM-5. Addiction. 2013;108(7):1186-1187. doi:10.1111/add.12162

8. King DL, Delfabbro PH. The cognitive psychology of Internet gaming disorder. Clinical psychology review. 2014;34(4):298-308. doi:10.1016/j.cpr.2014.03.006

9. Argyriou E, Davison CB, Lee TT. Response inhibition and internet gaming disorder: a meta-analysis. Addictive behaviors. 2017;71:54-60. doi:10.1016/j.addbeh.2017.02.026

10. Dong G, Hu Y, Lin X, Lu Q. What makes Internet addicts continue playing online even when faced by severe negative consequences? Possible explanations from an fMRI study. Biological psychology. 2013;94(2):282-289. doi:10.1016/j.biopsycho.2013.07.009

11. Saunders JB, Hao W, Long J, et al. Gaming disorder: Its delineation as an important condition for diagnosis, management, and prevention. Journal of behavioral addictions. 2017;6(3):271-279. doi:10.1556/2006.6.2017.039

12. Yao Y-W, Chen P-R, Chiang-shan RL, et al. Combined reality therapy and mindfulness meditation decrease intertemporal decisional impulsivity in young adults with Internet gaming disorder. Computers in Human Behavior. 2017;68:210-216. doi:10.1016/j.chb.2016.11.038

13. Stevens MW, King DL, Dorstyn D, Delfabbro PH. Cognitive-behavioral therapy for Internet gaming disorder: A systematic review and meta-analysis. Clinical psychology \& psychotherapy. 2019;26(2):191-203. doi:10.1002/cpp.2341

14. Zang Y, Jiang T, Lu Y, He Y, Tian L. Regional homogeneity approach to fMRI data analysis. Neuroimage. May 2004;22(1):394-400. doi:10.1016/j.neuroimage.2003.12.030

15. Liu J, Gao X, Osunde I, et al. Increased regional homogeneity in internet addiction disorder: a resting state functional magnetic resonance imaging study. Chin Med J. 2010;123(14):1904-1908.

. doi:10.3760/cma.j.issn.0366-6999.2010.14.014

16. Sun Y, Wang Y, Han X, et al. Sex differences in resting-state cerebral activity alterations in internet gaming disorder. Brain imaging behavior. 2019;13(5):1406-1417. doi:10.1007/s11682-018-9955-4

17. Lin X, Jia X, Zang Y-F, Dong G. Frequency-dependent changes in the amplitude of low-frequency fluctuations in internet gaming disorder. Frontiers in psychology. 2015;6:1471. doi:10.3389/fpsyg.2015.01471

18. Dong G, Huang J, Du X. Alterations in regional homogeneity of resting-state brain activity in internet gaming addicts. Behavioral Brain Functions. 2012;8(1):41. doi:10.1186/1744-9081-8-41

19. Yuan K, Jin C, Cheng P, et al. Amplitude of low frequency fluctuation abnormalities in adolescents with online gaming addiction. PLoS One. 2013;8(11)

20. Zhang J, Yao Y, Potenza MN, et al. Altered resting-state neural activity and changes following a craving behavioral intervention for Internet gaming disorder. Scientific Reports. 2016;6(1):1-8.

21. Han X, Wang Y, Jiang W, et al. Resting-state activity of prefrontal-striatal circuits in internet gaming disorder: changes with cognitive behavior therapy and predictors of treatment response. Frontiers in psychiatry. 2018;9:341. doi:10.3389/fpsyt.2018.00341 
22. Kuss DJ, Pontes HM, Griffiths MD. Neurobiological correlates in internet gaming disorder: A systematic literature review. Frontiers in psychiatry. 2018;9:166. doi:10.3389/fpsyt.2018.00166

23. Brand M, Young KS, Laier C. Prefrontal control and Internet addiction: a theoretical model and review of neuropsychological and neuroimaging findings. Frontiers in human neuroscience. 2014;8:375. doi:10.3389/fnhum.2014.00375

24. Mak KK, Lee K, Park C. Applications of machine learning in addiction studies: A systematic review. Psychiatry research. 2019;

25. Nielsen AN, Barch DM, Petersen SE, Schlaggar BL, Greene DJ. Machine Learning with Neuroimaging: Evaluating its Applications in Psychiatry. Biological Psychiatry: Cognitive Neuroscience and Neuroimaging. 2019;doi:10.1016/j.bpsc.2019.11.007

26. Norman KA, Polyn SM, Detre GJ, Haxby JV. Beyond mind-reading: multi-voxel pattern analysis of fMRI data. Trends in cognitive sciences. 2006;10(9):424-430.

27. Vieira S, Pinaya WH, Mechelli A. Using deep learning to investigate the neuroimaging correlates of psychiatric and neurological disorders: Methods and applications. Neuroscience \& Biobehavioral Reviews. 2017;74:58-75.

28. Wang Z, Dong H, Du X, Zhang JT, Dong GH. Decreased effective connection from the parahippocampal gyrus to the prefrontal cortex in Internet gaming disorder: A MVPA and spDCM study. J Behav Addict. Apr 1 2020;9(1):105-115. doi:10.1556/2006.2020.00012

29. Zhang J, Ma S, Yip SW, et al. Decreased functional connectivity between ventral tegmental area and nucleus accumbens in Internet gaming disorder: evidence from resting state functional magnetic resonance imaging. Behavioral and Brain Functions. 2015;11(1):37.

30. Ko C-H, Hsieh T-J, Wang P-W, et al. Altered gray matter density and disrupted functional connectivity of the amygdala in adults with Internet gaming disorder. Progress in Neuro-Psychopharmacology Biological Psychiatry. 2015;57(Progress in Neuro-Psychopharmacology):185-192.

31. Chen C-Y, Yen J-Y, Wang P-W, Liu G-C, Yen C-F, Ko C-H. Altered functional connectivity of the insula and nucleus accumbens in Internet gaming disorder: a resting state fMRI study. European addiction research. 2016;22(4):192-200. doi:10.1159/000440716

32. Bullmore E, Sporns O. Complex brain networks: graph theoretical analysis of structural and functional systems. Nature reviews neuroscience. 2009;10(3):186-198. doi:10.1038/nrn2575

33. Wang L, Wu L, Lin X, et al. Altered brain functional networks in people with Internet gaming disorder: Evidence from resting-state fMRI. Psychiatry Research: Neuroimaging. 2016;254:156-163. doi:10.1016/j.pscychresns.2016.07.001

34. Deshpande G, Sathian K, Hu X. Effect of hemodynamic variability on Granger causality analysis of fMRI. Neuroimage. 2010;52(3):884-896. doi:10.1016/j.neuroimage.2009.11.060

35. Roebroeck A, Formisano E, Goebel R. Mapping directed influence over the brain using Granger causality and fMRI. Neuroimage. 2005;25(1):230-242.

36. Dong G, Wang M, Wang Z, Zheng H, Du X, Potenza MN. Addiction severity modulates the precuneus involvement in internet gaming disorder: Functionality, morphology and effective connectivity. Progress in Neuro-Psychopharmacology Biological Psychiatry. 2020;98:109829. doi:10.1016/j.pnpbp.2019.109829

37. Yuan K, Qin W, Yu D, et al. Core brain networks interactions and cognitive control in internet gaming disorder individuals in late adolescence/early adulthood. Brain Structure Function. 2016;221(3):1427-1442. doi:10.1007/s00429-014-0982-7

38. Young K. Internet addiction: diagnosis and treatment considerations. Journal of Contemporary Psychotherapy. 2009;39(4):241-246. doi:10.1007/s10879-009-9120-x

39. Widyanto L, McMurran M. The psychometric properties of the internet addiction test. Cyberpsychology behavior. 2004;7(4):443-450. doi:10.1089/cpb.2004.7.443

40. Widyanto L, Griffiths MD, Brunsden V. A psychometric comparison of the Internet Addiction Test, the Internet-Related Problem Scale, and self-diagnosis. Cyberpsychology, Behavior, and Social Networking. 2011;14(3):141-149. doi:10.1089/cyber.2010.0151

41. Yan C-G, Wang X-D, Zuo X-N, Zang Y-F. DPABI: data processing \& analysis for (resting-state) brain imaging. Neuroinformatics. 2016;14(3):339-351. doi:10.1007/s12021-016-9299-4

42. Friston KJ, Williams S, Howard R, Frackowiak RS, Turner R. Movement-related effects in fMRI time-series. Magnetic resonance in medicine. 1996;35(3):346-355. doi:10.1002/mrm.1910350312

43. Biswal B, Zerrin Yetkin F, Haughton VM, Hyde JS. Functional connectivity in the motor cortex of resting human brain using echoplanar MRI. Magnetic resonance in medicine. 1995;34(4):537-541. doi:10.1002/mrm. 1910340409 
44. Zang Y, He Y, Zhu C, et al. Altered baseline brain activity in children with ADHD revealed by resting-state functional MRI. Brain Development. 2007;29(2):83-91. doi:10.1016/j.braindev.2006.07.002

45. Schrouff J, Rosa MJ, Rondina JM, et al. PRoNTo: pattern recognition for neuroimaging toolbox. Neuroinformatics. Jul 2013;11(3):319-37. doi:10.1007/s12021-013-9178-1

46. Chu C, Ni Y, Tan G, Saunders CJ, Ashburner J. Kernel regression for fMRI pattern prediction. NeuroImage. 2011;56(2):662-673. doi:10.1016/j.neuroimage.2010.03.058

47. Chu C, Mourao-Miranda J, Chiu YC, Kriegeskorte N, Tan G, Ashburner J. Utilizing temporal information in fMRI decoding: classifier using kernel regression methods. Neuroimage. Sep 15 2011;58(2):560-71. doi:10.1016/j.neuroimage.2011.06.053

48. Jollans L, Boyle R, Artiges E, et al. Quantifying performance of machine learning methods for neuroimaging data. Neuroimage. 2019;199:351-365. doi:10.1016/j.neuroimage.2019.05.082

49. Portugal LCL, Schrouff J, Stiffler R, et al. Predicting anxiety from wholebrain activity patterns to emotional faces in young adults: a machine learning approach. Neuroimage Clin. 2019;23:101813. doi:10.1016/j.nicl.2019.101813

50. Dosenbach NU, Nardos B, Cohen AL, et al. Prediction of individual brain maturity using fMRI. Science. 2010;329(5997):1358-1361.

51. He Y, Evans A. Graph theoretical modeling of brain connectivity. Current opinion in neurology. 2010;23(4):341-350. doi:10.1097/WCO.0b013e32833aa567

52. Barthelemy M. Betweenness centrality in large complex networks. The European physical journal B. 2004;38(2):163-168. doi:10.1140/epjb/e2004-00111-4

53. Latora V, Marchiori M. Efficient behavior of small-world networks. Physical review letters. 2001;87(19):198701. doi:10.1103/PhysRevLett.87.198701

54. dos Santos Siqueira A, Biazoli Junior CE, Comfort WE, Rohde LA, Sato JR. Abnormal functional resting-state networks in ADHD: graph theory and pattern recognition analysis of fMRI data. BioMed Research International. 2014;2014doi:10.1155/2014/380531

55. Wang J, Wang X, Xia M, Liao X, Evans A, He Y. GRETNA: a graph theoretical network analysis toolbox for imaging connectomics. Frontiers in human neuroscience. 2015;9:386.

56. Liao W, Wu G-R, Xu Q, et al. DynamicBC: a MATLAB toolbox for dynamic brain connectome analysis. Brain connectivity. 2014;4(10):780-790.

57. Lin X, Jia X, Zang Y, Dong G. Frequency-dependent changes in the amplitude of low-frequency fluctuations in internet gaming disorder. Frontiers in psychology. 2015;6:1471. doi:10.3389/fpsyg.2015.01471

58. Zheng H, Hu Y, Wang Z, Wang M, Du X, Dong G. Meta-analyses of the functional neural alterations in subjects with Internet gaming disorder: Similarities and differences across different paradigms. Progress in Neuro-Psychopharmacology Biological Psychiatry. 2019:109656. doi:10.1016/j.pnpbp.2019.109656

59. Walther A, Nili H, Ejaz N, Alink A, Kriegeskorte N, Diedrichsen J. Reliability of dissimilarity measures for multi-voxel pattern analysis. Neuroimage. 2016;137:188-200. doi:10.1016/j.neuroimage.2011.04.016

60. Desmurget M, Richard N, Harquel S, et al. Neural representations of ethologically relevant hand/mouth synergies in the human precentral gyrus. Proceedings of the National Academy of Sciences. 2014;111(15):5718-5722.

61. Graziano MS, Taylor CS, Moore T. Complex movements evoked by microstimulation of precentral cortex. Neuron. 2002;34(5):841-851. doi:10.1016/S0896-6273(02)00698-0

62. Zuo X-N, Ehmke R, Mennes M, et al. Network centrality in the human functional connectome. Cerebral cortex. 2012;22(8):1862-1875. doi:10.1093/cercor/bhr269

63. Luo X, Guo L, Dai X-J, et al. Abnormal intrinsic functional hubs in alcohol dependence: evidence from a voxelwise degree centrality analysis. Neuropsychiatric Disease Treatment. 2017;13:2011. doi:10.2147/NDT.S142742

64. Wiemeyer J, Hardy S. Serious games and motor learning: concepts, evidence, technology. Serious games and virtual worlds in education, professional development, and healthcare. IGI Global; 2013:197-220.

65. Radovanovic V. The influence of computer games on visual-motor integration in profoundly deaf children. British journal of special education. 2013;40(4):182-188.

66. Zhang Y, Mei W, Zhang JX, Wu Q, Zhang W. Decreased functional connectivity of insula-based network in young adults with internet 
gaming disorder. Experimental brain research. 2016;234(9):2553-2560. doi:10.1007/s00221-016-4659-8

67. Magno E, Simões-Franklin C, Robertson IH, Garavan H. The role of the dorsal anterior cingulate in evaluating behavior for achieving gains and avoiding losses. Journal of cognitive neuroscience. 2009;21(12):2328-2342.

68. Kim C, Kroger JK, Kim J. A functional dissociation of conflict processing within anterior cingulate cortex. Human brain mapping. 2011;32(2):304-312. doi:10.1002/hbm.21020

69. Knutson B, Westdorp A, Kaiser E, Hommer D. FMRI visualization of brain activity during a monetary incentive delay task. Neuroimage. 2000;12(1):20-27. doi:10.1006/nimg.2000.0593

70. Chun J-W, Park C-H, Kim J-Y, et al. Altered core networks of brain connectivity and personality traits in internet gaming disorder. Journal of Behavioral Addictions. 2020;doi:10.1556/2006.2020.00014

71. Lee D, Lee J, Namkoong K, Jung Y-C. Subregions of the anterior cingulate cortex form distinct functional connectivity patterns in young males with internet gaming disorder with comorbid depression. Frontiers in psychiatry. 2018;9:380. doi:10.3389/fpsyt.2018.00380

72. Zhang J, Dong H, Zhao Z, et al. Altered Neural Processing of Negative Stimuli in People with Internet Gaming Disorder: fMRI Evidence from the Comparison with Recreational Game Users. Journal of Affective Disorders. 2020; doi:10.1016/j.jad.2020.01.008

73. Sun Y, Ying H, Seetohul RM, et al. Brain fMRI study of crave induced by cue pictures in online game addicts (male adolescents). Behavioural brain research. 2012;233(2):563-576. doi:10.1016/j.bbr.2012.05.005

74. Goldstein RZ, Bechara A, Garavan H, Childress AR, Paulus MP, Volkow ND. The neurocircuitry of impaired insight in drug addiction. Trends in cognitive sciences. 2009;13(9):372-380. doi:10.1016/j.tics.2009.06.004

75. Wei Z, Yang N, Liu Y, et al. Resting-state functional connectivity between the dorsal anterior cingulate cortex and thalamus is associated with risky decision-making in nicotine addicts. Scientific reports. 2016;6:21778. doi:10.1038/srep21778 


\section{Figure Legends}

\section{Figure 1. Workflow of multi-voxel pattern analysis.}

The ReHo and ALFF map were calculated based on rs-fMRI data and then were entered into predictive

model as features to predict IGD severity. The KRR algorithm was applied to make prediction using

cross-validated approach. The model performance was assessed by permutation test. IAT: Internet

addiction test. 


\section{Figure 2. Performance of the model in predicting IAT score.}

Permutation distribution of the correlation coefficient ( $r$ ) and standard mean squared error (SMSE) for

the prediction analysis. The value obtained using the real scores are indicated by the red dash line. Higher

$r$ value and lower SMSE value indicate better performance of predictive models for ReHo and ALFF.

${ }^{*} p<0.05$ 


\section{Figure 3. Two selected ROIs and results of graph theory analysis.}

The threshold $T$ of network construction was set as 0.25 . (a) Two highly weighted ROIs in both predictive

models. (b) For the node of right precentral gyrus, the DC, BC, and NE are significantly related to IAT score.

(c) For the node of left postcentral gyrus, the DC, BC, and NE are not associated with IAT score. IAT:

Internet addiction test; DC: degree centrality; BC: betweenness centrality; NE: nodal efficiency. 


\section{Figure 4. Result of Granger causality analysis.}

(a) Two effective connectivity were associated to IAT score. (b) Scatter plot shows the positive correlation between IAT score and the connectivity from right precentral gyrus to left precentral gyrus $(r=0.160$,

p $<0.005$, GRF corrected). Each dot represents one subject. Semi-area represents $95 \%$ confidence interval for best-fit line. (c) Scatter plot shows the positive correlation between IAT score and the connectivity from right precentral gyrus to dACC $(r=0.158, p<0.005$, GRF corrected $)$. Each dot represents one subject. Semi-area represents $95 \%$ confidence interval for best-fit line. IAT: Internet addiction test; dACC: dorsal anterior cingulate cortex. 
bioRxiv preprint doi: https://doi.org/10.1101/2020.08.26.267989; this version posted August 27, 2020. The copyright holder for this preprint (which was not certified by peer review) is the author/funder, who has granted bioRxiv a license to display the preprint in perpetuity. It is made available under aCC-BY-NC-ND 4.0 International license.

\section{Table Legends}

Table 1. Participants demographics and IAT scores ( $\mathrm{N}=402)$.

\begin{tabular}{lcccc}
\hline & \multicolumn{2}{c}{ Male } & \multicolumn{2}{c}{ Female } \\
& Mean & SD & Mean & SD \\
\cline { 2 - 5 } & 21.78 & 2.67 & 21.07 & 1.98 \\
\hline Age (years) & 14.67 & 1.40 & 14.48 & 1.09 \\
Education (years) & 3.80 & 0.56 & 3.61 & 0.74 \\
Gaming history (years) & 6.94 & 3.00 & 7.08 & 3.59 \\
Gaming playing per weak(hours) & 49.57 & 16.55 & 51.44 & 14.85 \\
IAT score & &
\end{tabular}

SD: standard deviation; IAT: Internet addiction test. 
Table 2. Top 8 predictors for IGD severity and their relative weights in predictive power (percentage of the total weights) for predictive models based on ReHo and ALFF.

\begin{tabular}{|c|c|c|c|c|c|c|c|c|}
\hline & \multirow{2}{*}{$\begin{array}{c}\text { Serial } \\
\text { number }^{\mathrm{a}}\end{array}$} & \multirow{2}{*}{$\begin{array}{c}\text { Anatomical } \\
\text { region }{ }^{\mathrm{b}}\end{array}$} & \multicolumn{3}{|c|}{ MNI coordinates } & \multirow[t]{2}{*}{ Weight (\%) } & \multirow{2}{*}{$\begin{array}{c}\text { Size } \\
\text { (voxels) }\end{array}$} & \multirow{2}{*}{ Hemi } \\
\hline & & & $\mathrm{x}$ & $\mathrm{y}$ & $\mathrm{z}$ & & & \\
\hline \multirow[t]{8}{*}{$\begin{array}{l}\text { ReHo } \\
\text { model }\end{array}$} & 52 & Postcentral gyrus & -54 & -9 & 23 & 1.67 & 19 & $\mathrm{~L}$ \\
\hline & 75 & Postcentral gyrus & -38 & -27 & 60 & 1.57 & 19 & $\mathrm{~L}$ \\
\hline & 35 & Precentral gyrus & 60 & 8 & 34 & 1.46 & 17 & $\mathrm{R}$ \\
\hline & 137 & $\begin{array}{c}\text { Lateral occipital } \\
\text { cortex }\end{array}$ & 45 & -72 & 29 & 1.25 & 19 & $\mathrm{R}$ \\
\hline & 77 & Postcentral gyrus & -24 & -30 & 64 & 1.17 & 19 & $\mathrm{~L}$ \\
\hline & 62 & Precentral gyrus & -38 & -15 & 59 & 1.15 & 19 & $\mathrm{~L}$ \\
\hline & 87 & $\begin{array}{l}\text { Middle cingulate } \\
\text { cortex }\end{array}$ & 8 & -40 & 50 & 1.12 & 19 & $\mathrm{R}$ \\
\hline & 132 & Precuneus & 11 & -68 & 42 & 1.12 & 19 & $\mathrm{R}$ \\
\hline \multirow[t]{8}{*}{$\begin{array}{c}\text { ALFF } \\
\text { Model }\end{array}$} & 133 & Calcarine & 17 & -68 & 20 & 1.82 & 19 & $\mathrm{R}$ \\
\hline & 35 & Precentral gyrus & 60 & 8 & 34 & 1.63 & 17 & $\mathrm{R}$ \\
\hline & 147 & Calcarine & 9 & -76 & 14 & 1.56 & 19 & $\mathrm{R}$ \\
\hline & 52 & Postcentral gyrus & -54 & -9 & 23 & 1.44 & 19 & $\mathrm{~L}$ \\
\hline & 148 & Cuneus & 15 & -77 & 32 & 1.35 & 19 & $\mathrm{R}$ \\
\hline & 86 & Postcentral gyrus & 34 & -39 & 65 & 1.33 & 19 & $\mathrm{R}$ \\
\hline & 67 & $\begin{array}{c}\text { Supramarginal } \\
\text { gyrus }\end{array}$ & -54 & -22 & 22 & 1.23 & 19 & $\mathrm{~L}$ \\
\hline & 23 & $\begin{array}{c}\text { Inferior frontal } \\
\text { gyrus }\end{array}$ & -52 & 28 & 17 & 1.22 & 19 & $\mathrm{~L}$ \\
\hline
\end{tabular}

a: The serial numbers are taken from Dosenbach 160-node atlas.

b: The anatomical regions were defined based on Automated Anatomical Labeling (AAL) atlas. 
(a)

(b)

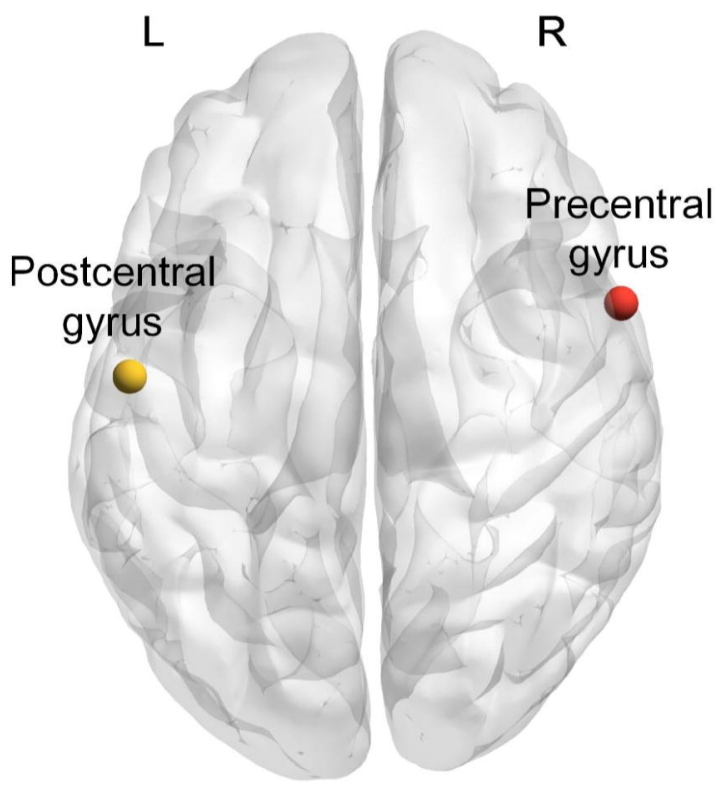

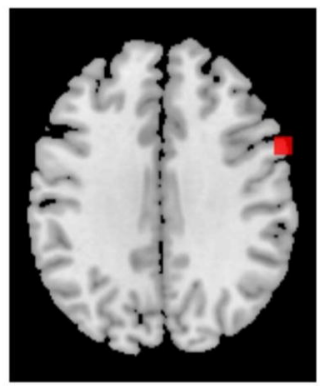

ROI 35: Right precentral gyrus
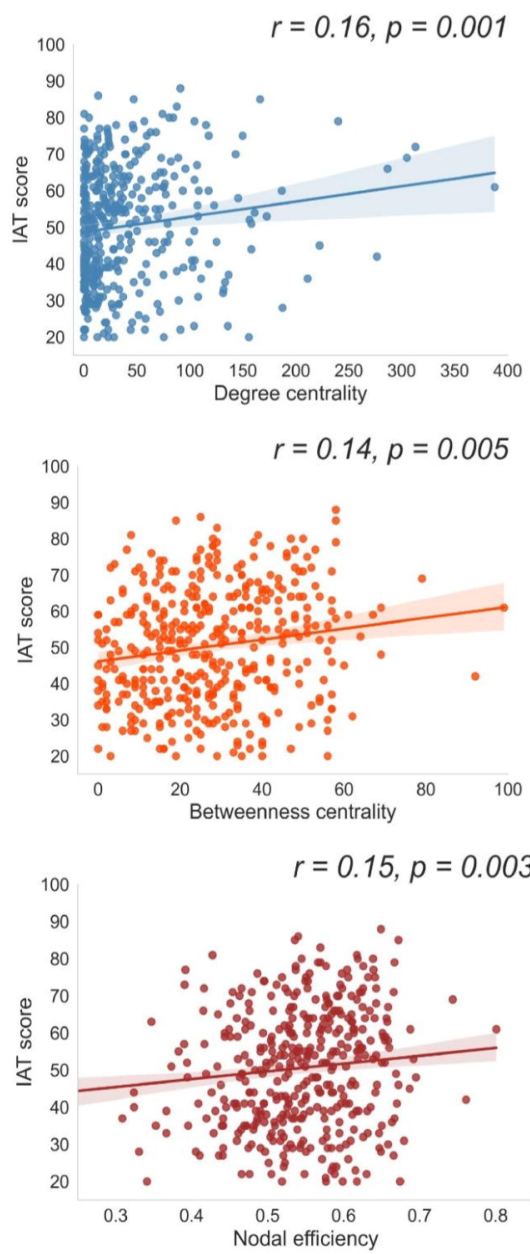

(c)

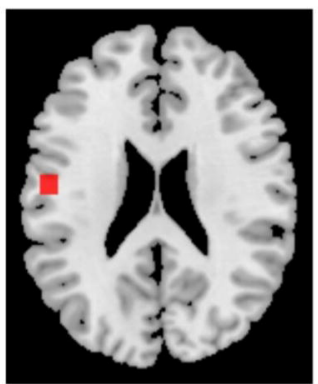

ROI 52: Left postcentral gyrus
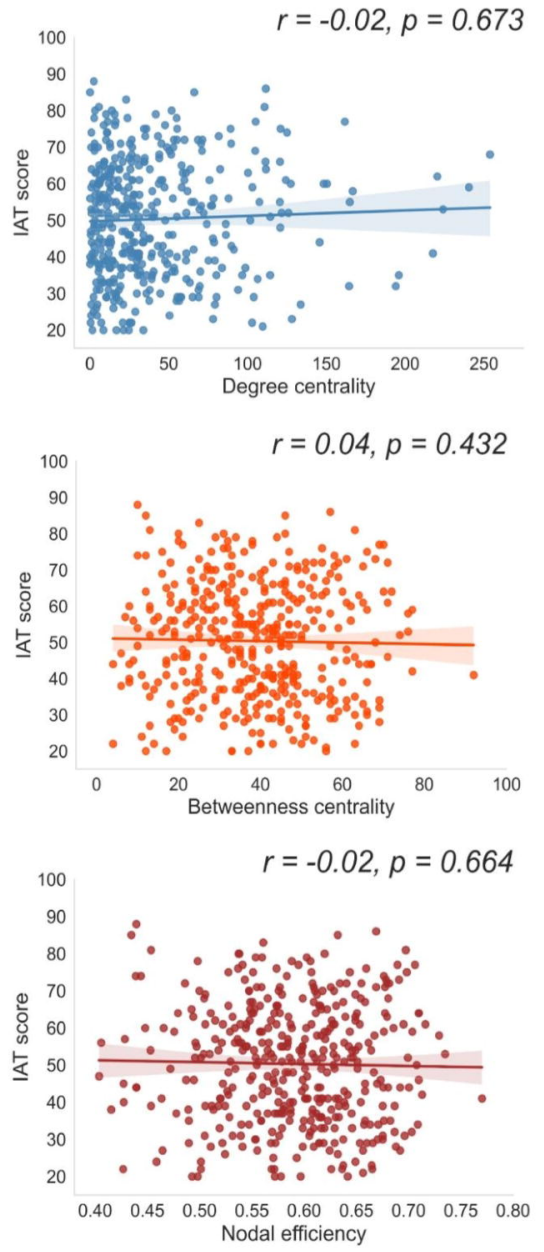
(a)

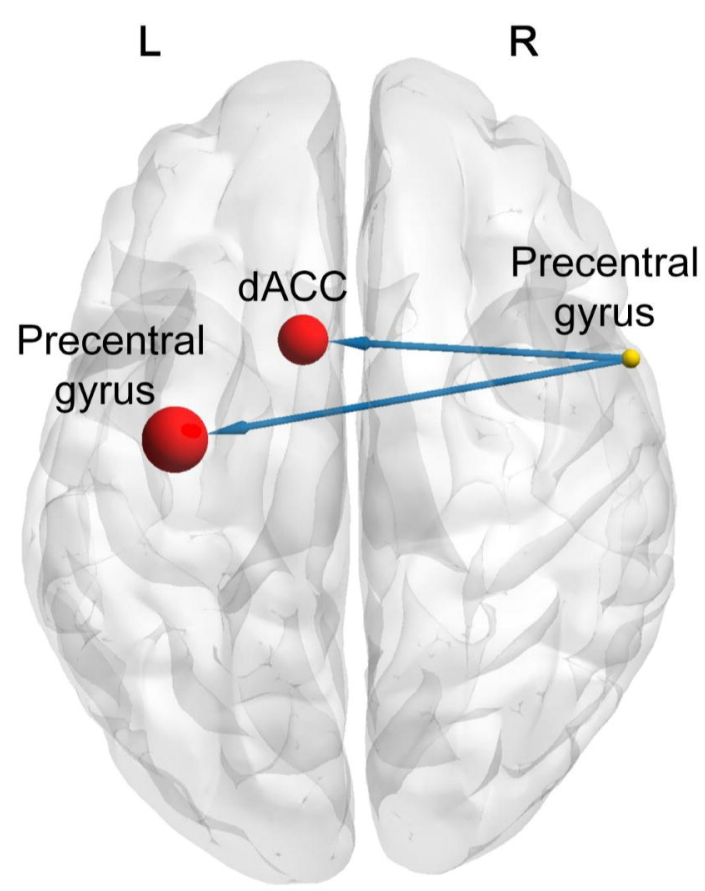

(b)
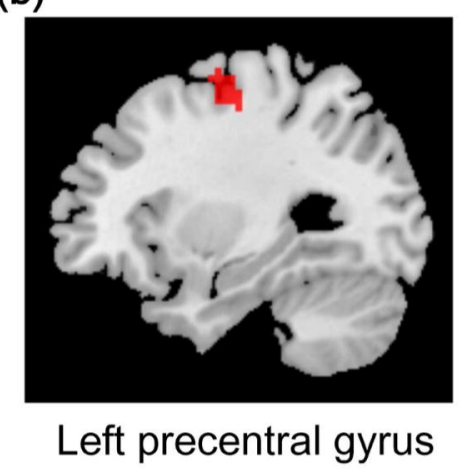

(c)

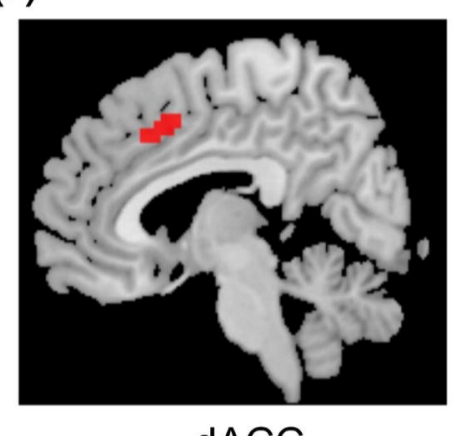

$$
r=0.160, p<0.005
$$

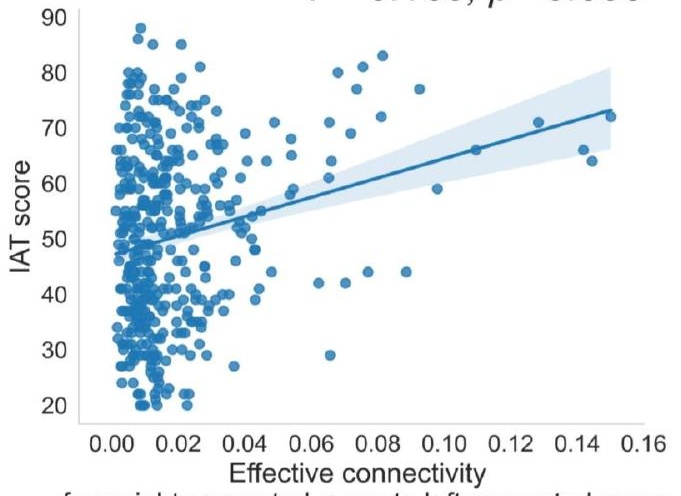
from right precentral gyrus to left precentral gyrus

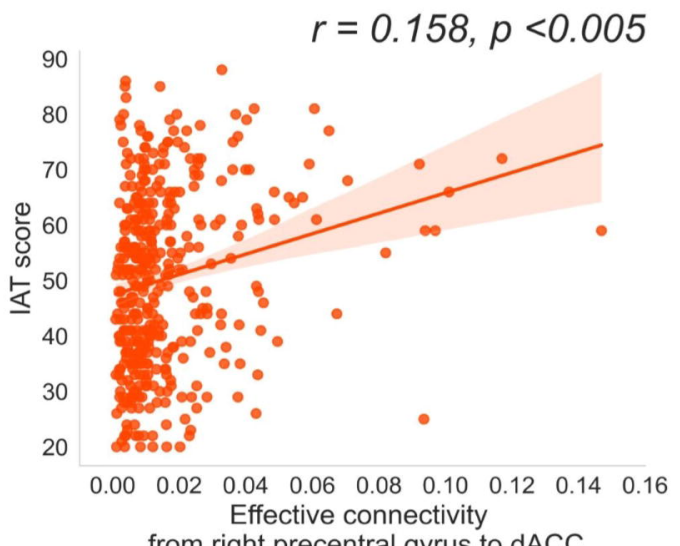
from right precentral gyrus to $\mathrm{dACC}$ 
fMRI data

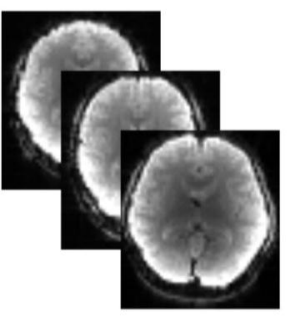

ReHo map

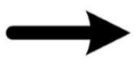

ALFF map

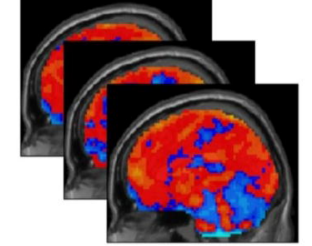

Features

ReHo

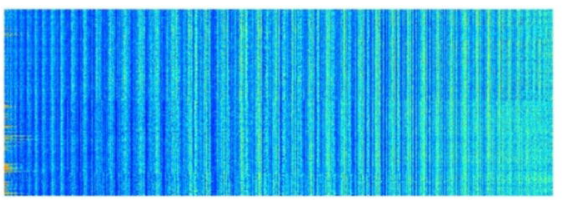

ALFF

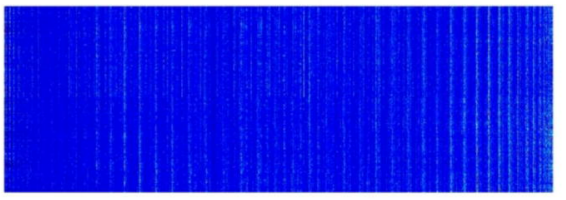

Enter features into Kernel Ridge Regression

Permutation test

Predictive model using ReHo value

Predictive model using ALFF value
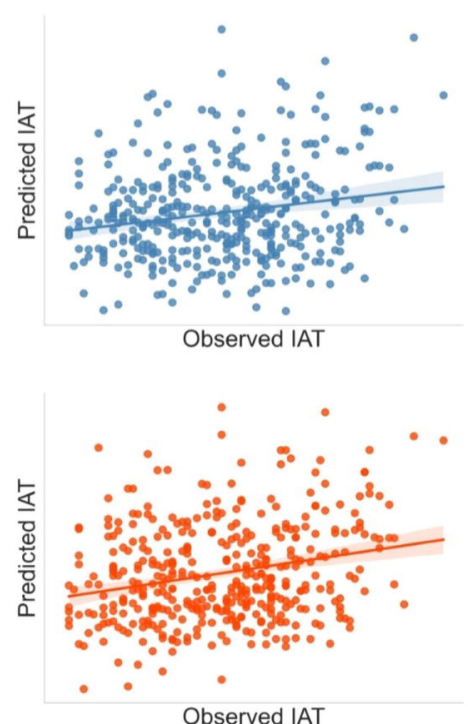
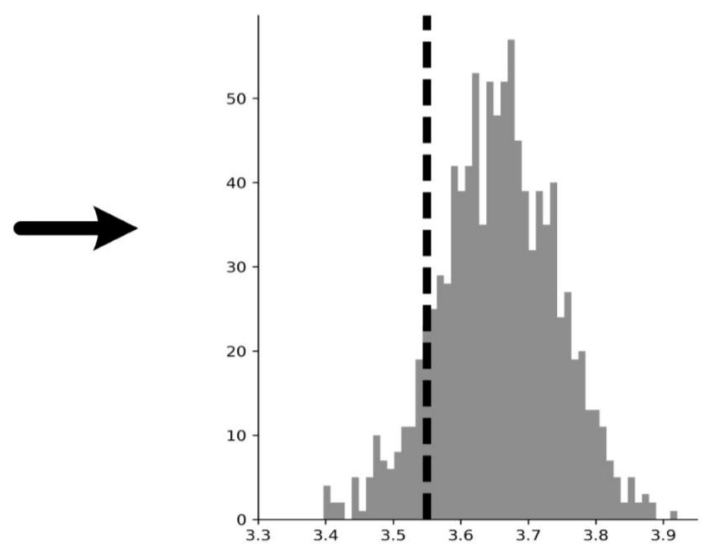


\section{ReHo}
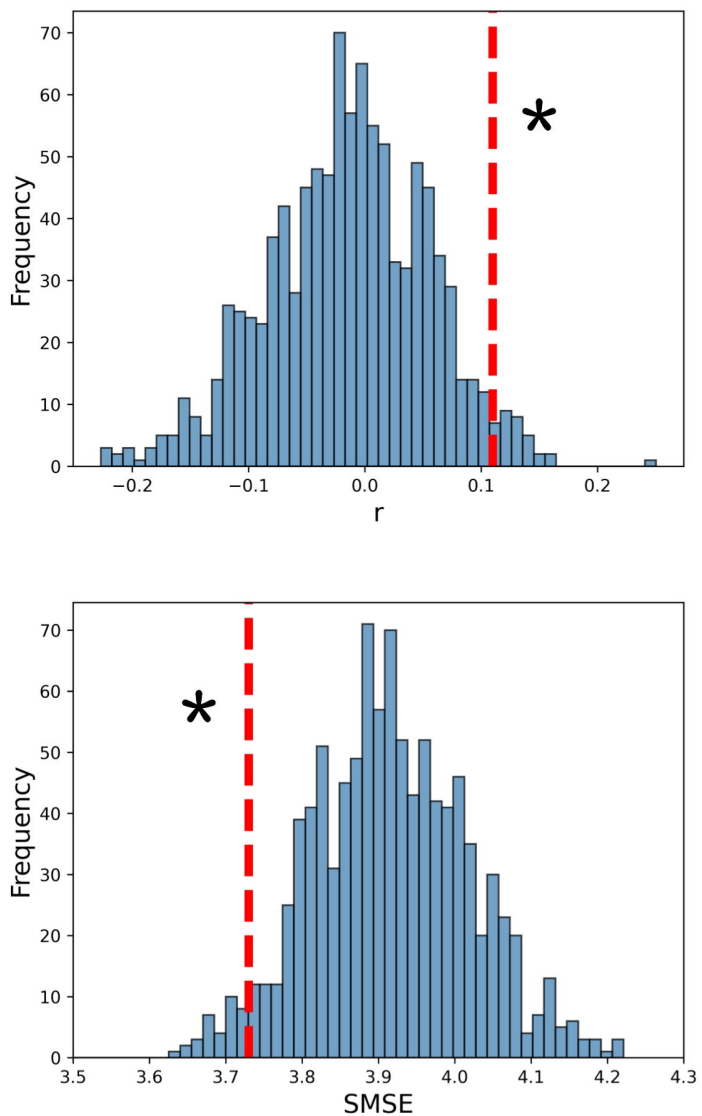

ALFF
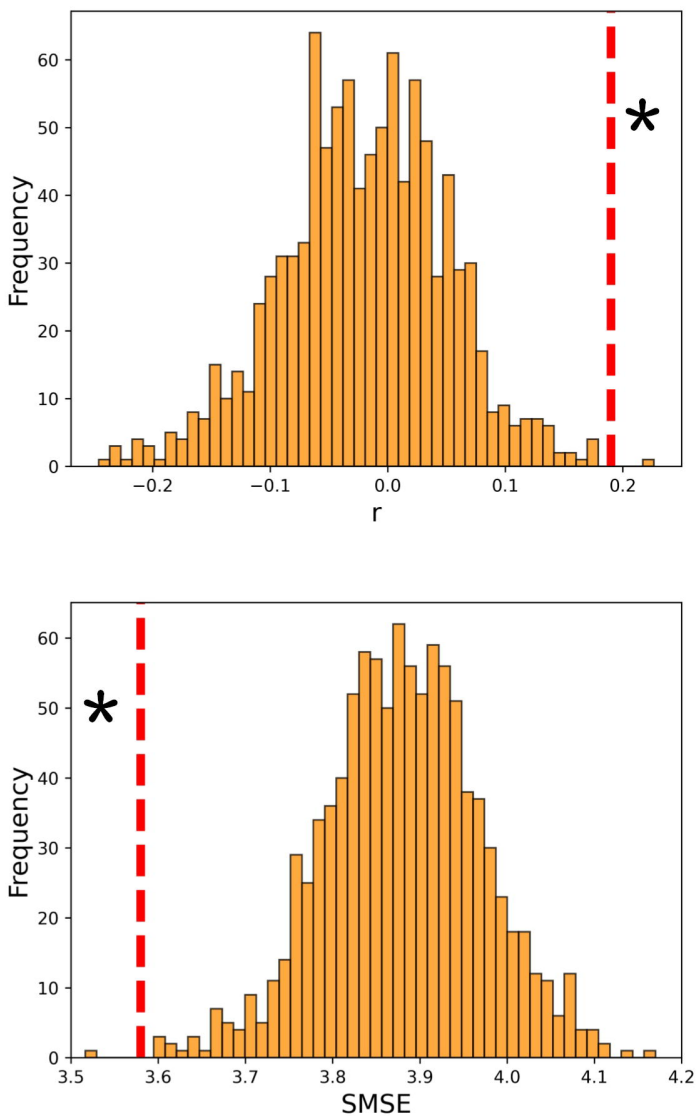